\title{
Was hilft bei komplizierter Trauer?
}

Fragestellung: Ist bei älteren Menschen mit komplizierter Trauer trauerspezifische Psychotherapie (Complicated Grief Treatment, CGT) wirksamer als interpersonelle Psychotherapie (IPT)?

Hintergrund: Verwitwung oder der Verlust von engen Bindungspersonen erhöht das Risiko von „komplizierter Trauer“, einem Muster psychischer Folgebeschwerden, die das psychosoziale Funktionsniveau und die physische Gesundheit der Betroffenen gravierend beeinträchtigen sowie das Suizidrisiko erhöhen. Bei Personen mittleren Alters wurde gezeigt, dass Trauersymptome weniger gut auf IPT ansprechen und eine Kombination aus Expositionsverfahren, IPT und motivierender Gesprächsführung erfolgversprechender ist. Eine Replikation dieser Ergebnisse wäre für den geriatrischen Bereich sehr interessant, da gegenüber Expositionstechniken im Alter Vorbehalte bestehen oder diese wegen der häufigen depressiven Symptomatik nicht erwogen werden.

Patienten und Methodik: Im Rahmen einer randomiserten kontrollierten Studie wurden 151 Personen rekrutiert. Einschlusskriterien waren ein Mindestalter von 50 Jahren und mindestens 30 Punkte auf dem Inventory of Complicated Grief (ICG). 74 Personen wurden auf CGT und 77 auf IPT randomisiert, die jeweils 16 Therapiesitzungen wurden wöchentlich durchgeführt. Wichtige Ziele der CGT sind die

Shear MK, Wang Y, Skritskaya $\mathrm{N}$ et al. Treatment of complicated grief in elderly persons. A randomized clinical trial. JAMA Psychiatry 2014; 71: 1287 - 95 Förderung eines normalen Trauerprozesses und der Abbau dysfunktionaler Faktoren wie Vermeidung. Imaginations- und Expositionsverfahren kommen dabei zum
Einsatz. Bei der IPT mit Fokus Trauer soll durch die Rekonstruktion der Beziehung und Umstände des Todes der gestörte Trauerprozess eingeleitet und eine Neuorientierung möglich werden. Primäre Erfolgsmaße waren die Response über die Clinical Global Impression (CGI) sowie die ICG-Responderraten. Weiterhin wurden konfundierende Variablen wie Alter, Geschlecht, Zeit nach dem Verlust, Güte der Beziehung, gewaltsamer Tod, antidepressive und anxiolytische Medikation, gegenwärtige depressive Episode, PTBS und MMS analysiert.

Ergebnisse: Die Teilnehmer waren im Schnitt 66 Jahre alt, $81 \%$ Frauen und mehr als $52 \%$ höher gebildet. Die Mehrheit erfüllte die Kriterien einer psychischen Störung (Depression 45,7\%, generalisierte Angststörung 24,5\%, PTBS 15,2\%, Panikstörung 11,9\%). Über die Hälfte der Teilnehmer hatte Suizidgedanken und erhielt eine antidepressive oder anxiolytische Medikation.

Die Remissionsraten (20 Wochen nach Therapiebeginn) lagen unter CGT deutlich höher (70,5\%) als in der IPT-Gruppe $(32,2 \%)$. Auch im CGI unterschieden sich beide Gruppen signifikant: Während in der IPT-Bedingung noch $64,1 \%$ als moderat krank eingeschätzt wurden, waren es bei CGT nur 35,2\%. Interessanterweise fand sich kein signifikanter Einfluss der vorgenannten Moderatorvariablen. Im 6-Monats-Follow-up blieben die Remissionsraten weitgehend stabil (CGT 100\%; IPT 86\%).

Schlussfolgerungen: CGT ist IPT statistisch wie klinisch überlegen und reduziert sowohl Trauer- als auch andere psychische Symptome. Alter, Diagnose, Medikation oder Güte der Beziehung haben darauf keinen Einfluss. Die Therapie wird von Älteren gut toleriert und rechtfertigt nicht die Zurückhaltung gegenüber Expositionsverfahren im Alter.

\section{- Kommentar von Petra Dykierek, Freiburg i. Br.}

\section{Auch trauernde Ältere profitieren von Expositionsverfahren}

Die Studie erfüllt mit einem JADAD-Score von 4 hohe methodische Ansprüche und ist klinisch von großer Relevanz. Infolge des demografischen Wandels ist mit einer Zunahme von Trauerstörungen zu rechnen, bei denen die bisher üblichen Psychound Pharmakotherapien nur begrenzte Erfolge vorweisen. Die Studie belegt, dass CGT mit ihren Expositionselementen nicht "schadet", sondern offenbar auch bei Älteren den Schlüssel zu einer adäquaten Trauerbewältigung darstellt. Sie sollte daher älteren Trauernden nicht vorenthalten werden. Eine Einschränkung der Studie besteht allerdings in der nicht repräsentativen Stichprobe. Mehrheitlich wurden weibliche, urbane, gebildete Menschen untersucht, ein Klientel, das ohnehin einen besseren Zugang zu psychotherapeutischer Unterstützung hat und nicht als "difficult to treat" gilt. Inwieweit die Ergebnisse auch auf Männer und/oder Betroffene mit niedrigen sozioökonomischen Status oder auf andere Kulturen übertragbar sind, lässt sich nicht eindeutig beantworten. In einer früheren Studie fanden sich allerdings keine Unterschiede zwischen afroamerikanischen und weißen Studienteilnehmern. Trotz dieser Einschränkung hat diese Studie eine wichtige Botschaft für Psychotherapie im Alter: Erlebnisvermeidung kann auch bei Älteren einen störungsaufrechterhaltenden Bedingungsfaktor darstellen, den es psychotherapeutisch aufzugreifen gilt.

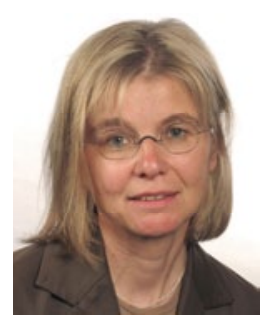

Dr. phil. Dipl.-Psych. Petra Dykierek, Freiburg i. Br.

Psychologische Psychotherapeutin, Abteilung für Psychiatrie und Psychotherapie, Universitätsklinikum Freiburg

E-Mail: petra.dykierek@uniklinik-freiburg.de 